\title{
The Spread of Ebola Virus Based On SEIHCR Model Zhaosheng Li
}

North China Electric Power University, Baoding 071000, China

1170188363@qq.com

\author{
Keywords: SEIHCR model, SEIR model
}

\begin{abstract}
The spread of virus is based on some mathematical models. An epidemic model SEIHCR was build up to take the special features of Ebola into consideration. These are treatments from hospital, infectious corpses and intensified contact tracing. This model is developed from the traditional SEIR model. The model's result, whose parameters are decided by using computer simulation, matches perfectly with the data reported by WHO, suggesting the validity of the improved model.
\end{abstract}

\section{Introduction}

Ebola virus disease, formerly known as Ebola haemorrhagic fever, is a severe, often fatal illness in humans. The current outbreak in West Africa, (first cases notified in March 2014), is the largest and most complex Ebola outbreak since the Ebola virus was first discovered in 1976. It started in Guinea and later spread across land borders to Sierra Leone and Liberia ${ }^{[1]}$. The analysis of the spread of epidemic has been a universal concern. Moreover, there have been a lot of research into the studies of the 2014 Ebola epidemic in West Africa ${ }^{[2]}$.

\section{The Traditional Epidemic Model}

The transmission of virus follows SEIR (susceptible-exposed-infections-recovered) dynamics and can be described by the following set of ordinary differential equations ${ }^{[3]}$ :

$$
\left\{\begin{array}{l}
\dot{S}(t)=-\beta S(t) I(t) / N \\
\dot{I}(t)=\sigma E(t)-\gamma I(t) \\
\dot{E}(t)=\beta S(t) I(t) / N-\sigma E(t) \\
\dot{R}(t)=\gamma I(t) \\
C \dot{U} M=\sigma E(t)
\end{array}\right.
$$

Where:

$S(t)$ is the number of susceptible individuals at time $t$,

$I(t)$ is the number of infectious individuals at time $t$,

$E(t)$ is the number of exposed individuals at time $t$,

$R(t)$ is the number of removed individuals at time $t$,

$C U M(t)$ is the cumulative number of Ebola cases from symptoms onset,

$N$ is the size of total population,

$1 / \gamma$ is the average duration of infectiousness.

$1 / \sigma$ is the average duration of incubation,

$\beta$ is the transmission rate per person day. $\beta$ is constant in absence of control interventions. However, $\beta$ is assumed to decay exponentially at rate $k^{[4]}$ after control measures are introduced at time $t \geq \tau$. That is,

$$
\beta(t)= \begin{cases}\beta_{0} & t<\tau \\ \beta_{0} e^{-k(1-\tau)} & t \geq \tau\end{cases}
$$




\section{The Improved Model}

The SEIR model is a basic model to predict the spread of disease. However, for the specific case of Ebola, we have to take other important factors into consideration. The things mainly considered are as follows.

1) The potential threat posed by infectious corpses and the provision of hospital care;

2) The involvement of contact tracing (CT).

Figure 1 shows a schematic presentation of the improved model SEIHCR, indicating the compartmental states and the transition rates among the states.

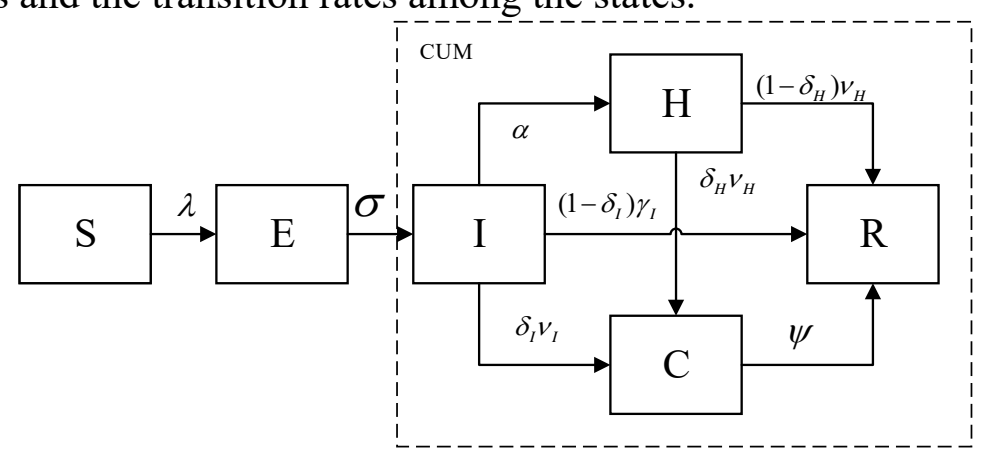

Figure 1. Compartmental flow of the SEICHR model

The traditional model SEIR should be modified ${ }^{[5]}$, and the improved SEIHCR(CT) model can be written as

$$
\left\{\begin{aligned}
\dot{S}(t)= & -\beta_{I} S(t) I(t) / N-\beta_{H} S(t) H(t) / N-\beta_{c} S(t) C(t) / N \\
\dot{E}(t)= & \beta_{I} S(t) I(t) / N+\beta_{H} S(t) H(t) / N+\beta_{c} S(t) C(t) / N-\sigma E(t) \\
& -\kappa(\alpha I(t)+\psi C(t)) \pi_{E} \omega_{E}(E(t) / N) \\
\dot{I}(t)= & \sigma E(t)-\alpha I(t)-\left(1-\delta_{1}\right) \gamma_{1} I(t)-\delta_{1} v_{1} I(t) \\
& -\kappa(\alpha I(t)+\psi C(t)) \pi_{I} \omega_{I}(I(t) / N) \\
\dot{H}(t)= & \alpha I(t)-\left(1-\delta_{H}\right) \gamma_{H} H(t)-\delta_{H} v_{H} H(t) \\
\dot{C}(t)= & \delta_{I} v_{I} I(t)+\delta_{H} v_{H} H(t)-\psi C(t) \\
\dot{R}(t)= & \left(1-\delta_{1}\right) \gamma_{1} I(t)+\left(1-\delta_{H}\right) \gamma_{H} H(t)+\psi C(t)
\end{aligned}\right.
$$

where:

$C(t)$ is the number of contaminated deceased at time $t$,

$H(t)$ is the number of hospitalized individuals at time $t$,

$\beta_{1}$ is the transmission rate outside hospital,

$\beta_{C}$ is the transmission rate due to improper handling of deceased, and

$$
\lambda=\beta_{I} \frac{I(t)}{N}+\beta_{H} \frac{H(t)}{N}+\beta_{C} \frac{C(t)}{N}
$$

$\beta_{H}$ is the transmission rate inside hospital,

$\lambda$ is the total transmission rate,

$\alpha$ is the rate of infectious individuals to be identified/isolated,

$1 / \gamma_{H}$ is the average time from symptoms onset to recovery inside hospital,

$1 / v_{H}$ is the average time from symptoms onset to death inside hospital,

$\delta_{H}$ is the fatality rate inside hospital,

$1 / \gamma_{I}$ is the average time from symptoms onset to recovery outside hospital,

$1 / v_{I}$ is the average time from symptoms onset to death outside hospital,

$\delta_{I}$ is the fatality rate outside hospital,

$1 / \psi$ is the average time until deceased is properly handled, 
$\kappa$ is the average number of contacts traced per identified/isolated infectious individual,

$\pi_{E}$ is the probability a contact traced exposed individual is isolated without causing a new case,

$\omega_{E}$ is the ratio probability that contact traced individual is infectious at time of originating case identification to the probability a random individual in the population is infectious.

Test the validity of the ultimate model, and compare the calculated data with the actual data. The cumulative number of individuals can be obtained by

$$
C U M(t+\Delta t)=C U M(t)+\int_{t}^{t+\Delta t} \sigma E(s) d s
$$

Equation (3) is improved from the original model SEIR and it is an ultimate model. Name it the SEIHCR model to illustrate its improvement and differences from the traditional SEIR model. The transition SEIR to SEIHCR shows how basic model can be greatly improved based on other factors and my understanding toward this problem.

\section{Determining Parameters in SEIHCR Model}

\subsection{Estimation of $R_{0}$}

In order to obtain the reproduction number $R_{0}$, a method is used. The method is as followes.

where:

$$
R_{0}=\rho\left(F V^{-1}\right)
$$

$\rho(A)$ denotes the spectral radius of a matrix $A$,

$F$ is the rate of appearance of new infections,

$V$ is the rate of transfer of individuals by all other means.

$F$ and $V$ that are corresponded with the SEIHCR model are

$$
F=\left(\begin{array}{l}
-\left(\beta_{I} S I / N+\beta_{H} S H / N+\beta_{C} S C / N\right) \\
\beta_{I} S I / N+\beta_{H} S H / N+\beta_{C} S C / N \\
0 \\
0 \\
0 \\
0
\end{array}\right), V=\left(\begin{array}{l}
0 \\
-\sigma E \\
\sigma E-\alpha I \\
\alpha I-\gamma_{H} H-v_{H} H \\
v_{I} I+v_{H} H-\psi C \\
\gamma_{I} I+\gamma_{H} H+\psi C
\end{array}\right)
$$

\subsection{Determination of other parameters}

The values of the parameters $\beta_{I}, \beta_{H}, \beta_{C}, \alpha$ and $\psi$ are estimated for the three countries using a least square curve fitting algorithm. The parameters $\sigma, \gamma_{I}, \gamma_{H}, v_{I}, v_{H}, \delta_{I}, \delta_{H}$ are borrowed from existing references ${ }^{[6]}$.

The basic reproduction number of the SEIHCR model is given by the following formula, computed by the next-generation method:

$$
R_{0}=\frac{\beta_{I}}{\alpha+\left(1-\delta_{I}\right) \gamma_{I}+\delta_{I} v_{I}}+\frac{\beta_{H}}{\left(1-\delta_{H}\right) \gamma_{H}+\delta_{H} v_{H}}+\frac{\beta_{C}}{\psi}
$$

Among all these parameters, $\beta_{I}$ is constant in absence of control interventions, just as $\beta$ from the SEIR model. After control measures are introduced at time $t \geq \tau, \beta_{I}$ is assumed to decay exponentially at rate $k^{[7]}$, That is,

$$
\beta_{I}(t)= \begin{cases}\beta_{I 0} & t<\tau \\ \beta_{I 0} e^{-k(1-\tau)} & t \geq \tau\end{cases}
$$

Since $k$ and $\gamma_{H}$ are both related to the efficacy of medicine, we assume their relationship to be

$$
\gamma_{H}=\theta k
$$

where $\theta$ is assumed to be 3.2 based on experience. 


\section{Result of the SEIHCR Model}

The dotted is used to represent the actual data of the cumulative numbers found in the WHO situation reports ${ }^{[8]}$ and the solid line to represent our calculated number from Equation (3) of the SEIHCR model. The start day of the simulation is defined as Day $1^{\text {st }}$.

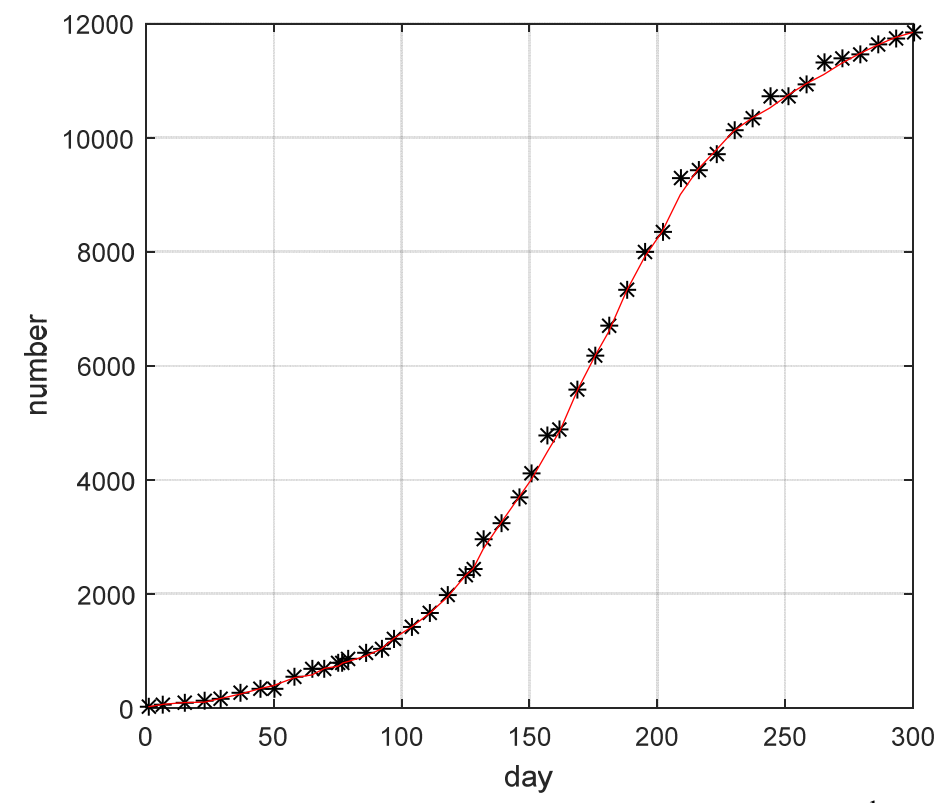

Figure 2. Simulation of cumulative cases in Sierra Leone from May $27^{\text {th }}, 2014$ to Feb $4^{\text {th }}, 2015$. The parameter values are $N=6000000, \beta_{I 0}=0.31, \beta_{H}=0.0001, \beta_{C}=0.125, \alpha=0.2, \gamma_{I}=1 / 21$, $v_{I}=1 / 8, v_{H}=1 / 31, \psi=1 / 5.5, \kappa=0, k=0.015, \tau=160$

These simulations are accomplished by varying combinations of parameters. The calculated data become more smoothly and closely approximate to the actual reported data with the simulation for Sierra Leone being the perfect approximation

\section{References}

[1] World Health Organization, Ebola Virus disease fact sheets, update Sep 2014, http : // www. who. Int / mediacentre /fact sheets /fs103/en/

[2] Chowell, G., Nishiura, H., Transmission dynamics and control of Ebola virus disease (EVD): a review, BMC Medicine, 12(196):1-16, 2014.

[3] Chowell, G., Hengartner, N. W., Castillo-Chavez, C., Fenimore, P. W., Hyman, J.M. The basic reproductive number of Ebola and the effects of public health measures: the cases of Congo and Uganda, Journal of Theoretical Biology, 229: 119-126, 2004.

[4] Lekone, P. E., Finkenstädt, B. F., Statistical inference in a stochastic epidemic SEIR model with control intervention: Ebola as a case study, Biometrics, 62(4): 1170-1177, 2006.

[5] Rivers, C. M., Lofgren, E.T., Marathe, M., Eubank, S., Lewis, B.L., Modeling the Impact of Interventions on an Epidemic of Ebola in Sierra Leone and Liberia. PLOS Currents Outbreaks. Oct 17th, 2014.

[6] Althaus, C. L., Estimating the reproduction number of Ebola virus (EBOV) during the 2014 outbreak in West Africa, PLOS Currents Outbreaks, Sep 2nd, 2014.

[7] Lekone, P. E., Finkenstädt, B. F., Statistical inference in a stochastic epidemic SEIR model with control intervention: Ebola as a case study, Biometrics, 62(4): 1170-1177, 2006.

[8] World Health Organization, Ebola situation report, http://www.who.int/csr/disease/ebola/situation-reports/en/. 